\title{
Semi-empirical calibration of the Integral Equation Model for SAR data in C-band and cross polarization using radar images and field measurements
}

\author{
Nicolas BAGHDADI ${ }^{1}$, Jad ABOU CHAAYA ${ }^{1}$, and Mehrez ZRIBI ${ }^{2}$ \\ ${ }^{1}$ CEMAGREF, UMR TETIS, 500 rue François Breton, 34093 Montpellier cedex 5, France \\ ${ }^{2}$ IRD-CESBIO, 18 av. Edouard Belin, BP 2801, 31401 Toulouse cedex 9, France \\ E-mail: nicolas.baghdadi@teledetection.fr \\ Tel.: 334675487 24; Fax: 33467548700
}

\begin{abstract}
The estimation of surface soil parameters (moisture and roughness) from Synthetic Aperture Radar (SAR) images requires the use of well-calibrated backscattering models. The objective of this paper is to extend the semi-empirical calibration of the backscattering Integral Equation Model (IEM) initially proposed by Baghdadi et al. (2004 and 2006) for $\mathrm{HH}$ and VV polarizations to HV polarization. The approach consisted in replacing the measured correlation length by a fitting/calibration parameter so that model simulations would closely agree with radar measurements. This calibration in C-band covers radar configurations with incidence angles between $24^{\circ}$ and $45.8^{\circ}$. Good agreement was found between the backscattering coefficient provided by the SAR and that simulated by the calibrated version of the IEM.
\end{abstract}

Index Terms - Integral Equation Model (IEM), calibration, SAR images

\section{INTRODUCTION}

E stimation of soil surface characteristics (SSC) from SAR images is generally performed using either empirical or physical approaches. The empirical approach is based on experimental data, in order to establish relationships linking the radar backscattering coefficient to SSC and to SAR sensor parameters (radar wavelength, incidence angle, polarization). For bare soils, the radar backscattering coefficient $\left(\sigma^{\circ}\right)$ follows an exponential relationship with the soil surface roughness, and increases linearly or exponentially with the volumetric soil moisture for values between approximately 5 and $40 \mathrm{~cm}^{3} / \mathrm{cm}^{3}$ (e.g. [1], [2], [3], [4]). These relationships are often inapplicable to study sites other than those on which they were established, which restrict their use. Moreover, the collection of a database that is representative of different physical conditions of soil surface is extremely difficult under various SAR sensor parameters.

Numerous semi-empirical models for estimating both soil moisture and surface roughness from radar backscattering coefficients have been reported in the literature. The most popular are those developed by Oh [5] and Dubois et al. [6]. Discrepancies with experimental measurements in agricultural areas were observed in several studies (e.g. [7] and [8]).

The physical approach uses a backscattering model capable of reproducing the radar signal from SAR parameters and soil surface characteristics (soil moisture, surface roughness, and soil composition for bare soils). The Integral Equation Model (IEM: [9]) is one of the physical models most widely used in inversion procedures of SAR images for retrieving soil moisture and/or roughness parameters ([3], [10]). However, conflicting results have been obtained. Some studies have shown good agreement between measured backscattering coefficients and those predicted by the models (e.g. [10] and [11]), while others have found large differences between simulations and measurements, rendering the inversion results inaccurate between them (e.g. [7], [8], [12], [13], [14]).

The description of surface roughness on bare soils in the IEM is currently based on three parameters [9]: the correlation function, the correlation length, and the standard deviation of heights (s). A number of studies have shown that the backscattering coefficient varies considerably depending on the shape of the correlation function, and that the measurements of correlation length are inaccurate (highly dependent on length and number of roughness profiles), which introduces significant errors into the modelled radar signal ([15], [16], [17]).

Baghdadi et al. ([13] and [14]) proposed an empirical calibration of the IEM for $\mathrm{HH}$ and VV polarizations. It is based on a large experimental database composed of SAR images and ground measurements of soil moisture and surface roughness. In this calibration, the discrepancies observed between the IEM and the SAR data were related both to the shape of the correlation function and the accuracy of the correlation length measurements. The other physical input parameters used in the IEM as standard deviation of heights and soil moisture are assumed to be relatively accurate. The approach consisted of replacing the measured correlation length, for each SAR configuration (radar wavelength, incidence angle, and polarization), by a fitting/calibration parameter (Lopt), so that the IEM model reproduce better the radar backscattering coefficient. It replaces the inaccurate correlation length and calibrates empirically the model. Calibration results showed good agreement between the backscattering coefficients given by the SAR sensors and those estimated from the calibrated IEM for data in C-band, $\mathrm{HH}$ and VV polarizations, and incidence angles between $20^{\circ}$ 
and $48^{\circ}$. The calibration parameter is found dependent on $r m s$ surface height, radar wavelength, polarization, and incidence angle.

In this study, we intend to extend the calibration of the IEM model to SAR data in cross-polarization.

\section{DATABASE}

\section{A. Study areas}

Simultaneously with several SAR acquisition campaigns over four study sites in France and Italy (Table 1), field measurements of soil moisture and surface roughness have been achieved.

\section{B. Satellite data}

C-band SAR images were obtained using ASAR and SIR-C sensors. The radar data are available in HV polarization with incidences between $24^{\circ}$ and $45.8^{\circ}$. Images were first calibrated to enable the derivation of backscattering coefficients $\left(\sigma^{\circ}\right)$. The average backscattering coefficient was then calculated for each reference field. The standard deviation of ASAR backscattering coefficient on the field scale is around $1 \mathrm{~dB}$.

TABLE I

SAR CONFIGURATIONS OF THE DATABASE.

\begin{tabular}{|c|c|c|c|}
\hline Site & $\begin{array}{l}\text { SAR } \\
\text { sensor }\end{array}$ & Year & Polarization and incidence \\
\hline $\begin{array}{l}\text { Villamblain } \\
\text { [14] }\end{array}$ & ASAR & 2004,2003 & $\mathrm{HV}-24^{\circ}, 34^{\circ}, 37^{\circ}, 40^{\circ}, 41^{\circ}, 43^{\circ}$ \\
\hline Touch [14] & ASAR & 2004 & 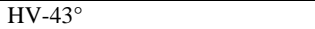 \\
\hline Orgeval [7] & SIR-C & 1997 & $\mathrm{HV}-44^{\circ}$ \\
\hline Matera [12] & SIR-C & 1994 & $\mathrm{HV}-45.8^{\circ}$ \\
\hline
\end{tabular}

\section{Field data}

Soil moisture and surface roughness measurements were carried out on several bare soil reference fields. The volumetric water content at field scale was assumed to be equal to the mean value estimated from several samples collected from the top $5 \mathrm{~cm}$ of soil using the gravimetric method and a TDR (Time Domain Reflectometry) probe. The soil moistures range from 5 to $47 \mathrm{~cm}^{3} / \mathrm{cm}^{3}$ with a standard deviation of about $5 \mathrm{~cm}^{3} / \mathrm{cm}^{3}$.

One and three meter long roughness profiles have been recorded parallel and perpendicular to the row direction. Ten roughness profiles were sampled for each training field. From these measurements, the two roughness parameters, root mean square surface height $(s)$ and correlation length $(L)$, were calculated using the mean of all correlation functions. The rms surface heights range from $0.6 \mathrm{~cm}$ to $3.7 \mathrm{~cm}$.

\section{IEM BACKSCATTERING MODEL}

The Integral Equation Model [9] has a validity domain that covers the range of roughness values that are commonly encountered for agricultural surfaces $(k . s \leq 3$, where $k$ is the wave number $\cong 1.11 \mathrm{~cm}^{-1}$ for a frequency in C-band of 5.3 $\mathrm{GHz}$ ). Over bare soils in agricultural areas, the backscattering coefficient of the surface contribution is expressed for cross polarization as:

$$
\begin{aligned}
& \sigma_{h v}^{\circ}=\frac{k^{2}}{16 \pi} e^{-2 k^{2} s^{2} \cos ^{2} \theta} \sum_{n=1}^{+\infty} \sum_{m=1}^{+\infty} \frac{\left(k^{2} s^{2} \cos ^{2} \theta\right)^{n+m}}{n ! m !} \\
& \iint\left[\left.F_{h v}(u, v)\right|^{2}+F_{h v}(u, v) F_{h v}^{*}(-u,-v)\right] W^{(n)}(u-k \sin \theta, v) W^{(m)}(u+k \sin \theta, v) d u d v
\end{aligned}
$$

Where:

$$
\begin{aligned}
& F_{h v}(u, v)=\frac{u v}{k \cos \theta}\left[\frac{8 R^{2}}{\sqrt{k^{2}-u^{2}-v^{2}}}+\frac{-2+6 R^{2}+\frac{(1+R)^{2}}{\varepsilon_{r}}+\varepsilon_{r}(1-R)^{2}}{\sqrt{\varepsilon_{r} k^{2}-u^{2}-v^{2}}}\right] \\
& R=\frac{R_{v}-R_{h}}{2} \\
& R_{h}=\frac{\mu_{r} \cos \theta-\sqrt{\mu_{r} \varepsilon_{r}-\sin ^{2} \theta}}{\mu_{r} \cos \theta+\sqrt{\mu_{r} \varepsilon_{r}-\sin ^{2} \theta}} \text { : Fresnel coefficient at horizontal }
\end{aligned}
$$

polarization

$$
R_{v}=\frac{\varepsilon_{r} \cos \theta-\sqrt{\mu_{r} \varepsilon_{r}-\sin ^{2} \theta}}{\varepsilon_{r} \cos \theta+\sqrt{\mu_{r} \varepsilon_{r}-\sin ^{2} \theta}}: \text { Fresnel coefficient at vertical }
$$
polarization

$\varepsilon_{r}$ : dielectric constant obtained using the empirical model of Hallikaïnen et al. [18].

$\mu_{r}$ : relative permeability

$\theta$ : incidence angle

Re: real part of the complex number

$f_{p p}^{*}$ : conjugate of the complex number $f_{p p}$

$W^{(n)}$ is the Fourier transform of the $n$th power of the surface correlation coefficient:

$$
W^{(n)}(a, b)=\frac{1}{2 \pi} \iint \rho^{n}(x, y) e^{-i(a x+b y)} d x d y
$$

$\rho(x, y)$ : surface correlation function. Its distribution is exponential for low surface roughness values and Gaussian for high surface roughness values:

$$
\begin{aligned}
\rho(x, y)=e^{-\frac{|x|+|y|}{L}} & : \text { exponential } \\
=e^{-\frac{x^{2}+y^{2}}{L^{2}}}: & \text { Gaussian }
\end{aligned}
$$

Values of $\sigma^{\circ}$ simulated by IEM using the correlation length measurements, and $\sigma^{\circ}$ calculated from SAR images were compared (Fig. 1). The mean and the standard deviation of the difference between $\sigma^{\circ}$-IEM and $\sigma^{\circ}$-SAR were calculated using 193 data (Villamblain and Touch databases). The IEM in $\mathrm{HV}$ polarization overestimates the $\sigma^{\circ}$-SAR of about $18.6 \mathrm{~dB}$ with the exponential function and underestimates it of $-19.4 \mathrm{~dB}$ with the Gaussian function regardless of the incidence angle used. With both the exponential and the Gaussian correlation functions, the standard deviation of the error is very high (11.5 and $21.4 \mathrm{~dB}$, respectively).

These results led to the conclusion that the IEM simulations do not correctly fit SAR measurements, regardless of the correlation function used. The poor correlation noted between IEM and SAR has nothing to do with the IEM's validity domain. The mismatch noted between IEM simulations and SAR data is related to the uncertainty of the correlation length measurements and to the model itself [14]. Indeed, according to Oh and Kay [16], correlation length measurements are 
unreliable when conventional profilometers of 1 or $2 \mathrm{~m}$ long are used (error over 50\%).

In the following paragraph, we propose a semi-empirical calibration of the IEM by redefining the measured correlation length so as to ensure better agreement between the model and the data.

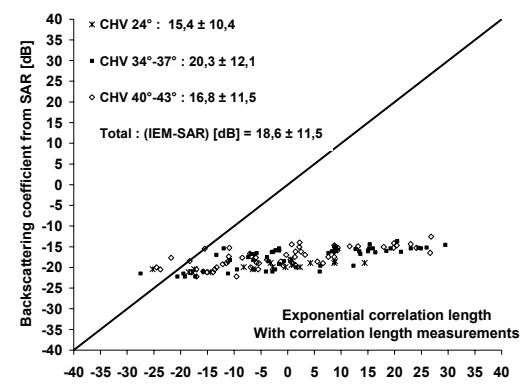

Backscattering coefficient from IEM [dB]

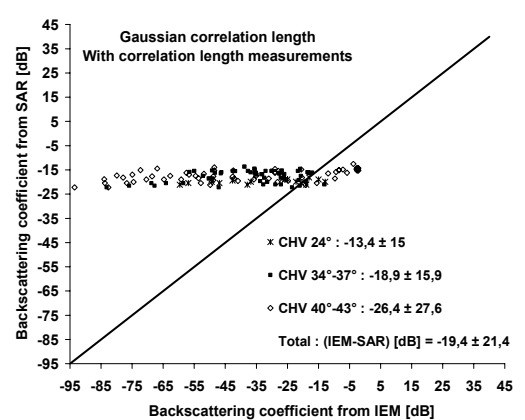

Fig. 1. Comparison between $\sigma^{\circ}$-IEM using correlation length measurements, and $\sigma^{\circ}$-SAR. Mean and standard deviation of the difference were calculated.

\section{EMPIRICAL CALIBRATION OF THE IEM}

The objective is to propose a robust calibration of the IEM model that would allow reproducing correctly the SAR signal. The approach consists of replacing the measured correlation length, which is not only the least accurate of model input parameters but also the most difficult to measure, by a calibration parameter (Lopt). For each element of the experimental database, Lopt ensures a good fit between IEM simulation and SAR data. Large database consisting of Cband SAR images (ASAR) and ground measurements soil moisture and surface roughness was used. Figure 2 shows the behaviour of the IEM as a function of the correlation length. It shows that Lopt has two possible solutions, Lopt1 and Lopt2, which ensure good agreement between the IEM and the SAR backscattering coefficient. The difference between Lopt1 and Lopt2 is smaller with Gaussian function than with exponential one. Lopt1 for Gaussian and exponential functions remain similar for low and medium rms surface heights, and become larger with exponential function for high rms height. However, Lopt2 obtained with exponential function are very different from those with Gaussian function for different rms height values. Only Villamblain and Touch databases were used in this calibration phase.

Figures 3 and 4 show that the calibration parameters Lopt1 and Lopt2 depend for each correlation function on rms surface height and incidence angle. They show that the calibration parameter Lopt1 decreases slightly with $s$ for the exponential correlation function; whereas for the Gaussian function it decreases slightly for $s<1 \mathrm{~cm}$ and then increases slightly with the $s$. Moreover, Lopt2 increases with $s$ for both correlation functions. Results also showed a slight dependence between Lopt and incidence angle.

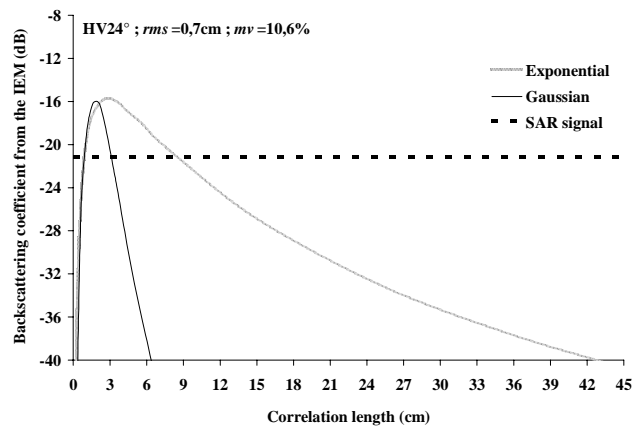

Fig. 2. Behaviour of the IEM as a function of correlation length (C-HV).

The two apparently independent parameters $r m s$ height and correlation length are in fact related. Davidson et al. [19] observed over agricultural surfaces a linear relation between $r m s$ height and $L$ for a wide range of roughness conditions.

\section{PARAMETERIZATION OF THE CALIBRATION PARAMETER}

When Lopt1 (the lowest value) was used in the IEM model, it proved difficult for some radar configurations to ensure the correct physical behaviour between $\sigma^{\circ}$ and the $s$ for both exponential and Gaussian correlation functions, especially those with low incidence angles. $\sigma^{\circ}$-IEM decreases from $s \sim 1 \mathrm{~cm}$ instead of continuing to increase. Only Lopt2 (the highest value) with Gaussian correlation function ensures a correct physical behaviour of $\sigma^{\circ}$ as a function of $s$ (increasing $\sigma^{\circ}$ with increasing $s$, for a given moisture value). In the fitting process, we added the few points with ks $>3$ with the objective to analyze the behaviour of fitting parameter for surface roughness slightly at outside of the IEM validity domain (svalues between 3 and $3.6 \mathrm{~cm}$ ). Results show that the fitting parameter follows the same relationship for s between $0.6 \mathrm{~cm}$ and $3 \mathrm{~cm}$ and between $0.6 \mathrm{~cm}$ and $3.6 \mathrm{~cm}$.

For Gaussian correlation function, Lopt2 follows a linear relationship (C-HV):

$$
\operatorname{Lopt} 2(s, \theta)=\alpha+\beta s
$$

$\beta$ is dependent of incidence angle. Lopt2 and $s$ are in $\mathrm{cm}$.

Next, we calculated the expression of Lopt2 as a function of rms surface height and incidence angle $(\theta)$ :

$$
\text { Lopt } 2(s, \theta)=0.9157+1.2289(\sin 0.1543 \theta)^{-0.3139} s \quad(2
$$

The coefficient of determination $R^{2}$ is 0.96 .

Results provided by the IEM after calibration, using Lopt2 given by the equation (2), show a small difference between calibrated IEM simulations and SAR data. Bias and standard deviation of the error have decreased from $-19.4 \mathrm{~dB}$ to +0.3 $\mathrm{dB}$, and from $21.4 \mathrm{~dB}$ to $2.3 \mathrm{~dB}$, respectively. Moreover, the parameterization of the calibration parameter thus enables a 
correct simulation of the backscattering signal. A correct physical behaviour between the backscattering coefficient and the rms surface height was observed for different incidence angles and soil moisture (Fig. 5). The expression of Lopt2 (eq. (2)) was validated for incidences between $22^{\circ}$ and $50^{\circ}$. The use of Gaussian correlation function and the expression of Lopt2 ensures correct physical behaviour of IEM to approximately $s=4 \mathrm{~cm}$ (C-band; HV).
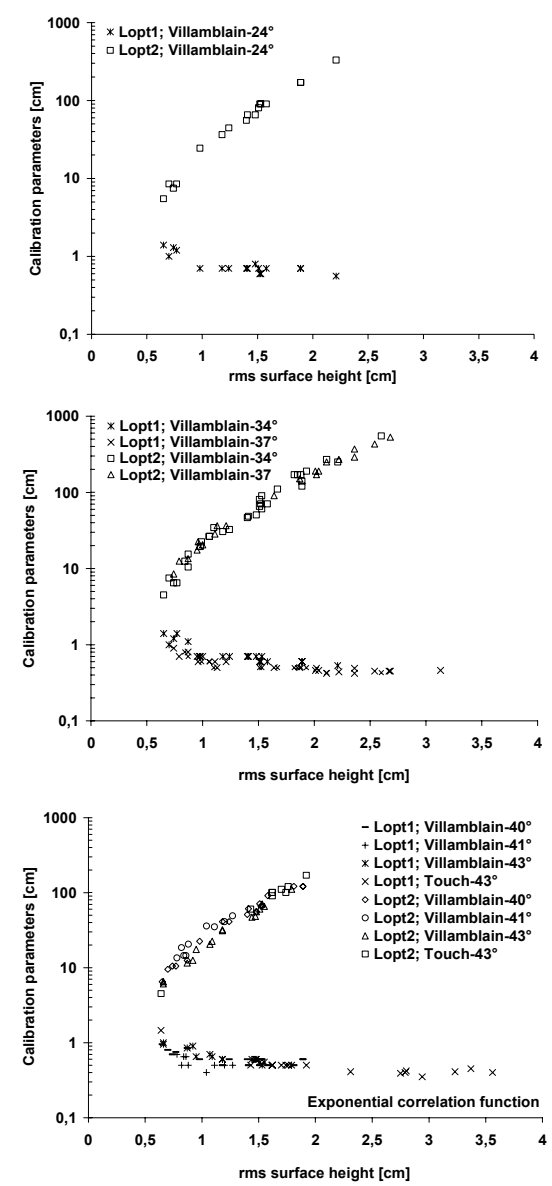

Fig. 3. Calibration parameters Lopt1 and Lopt2 as a function of $s$ (C-band, $\mathrm{HV}$ ), with exponential correlation function.

With this fitting parameter (Lopt2), the correlation function can be considered as Gaussian for all $r m s$ values $(<4 \mathrm{~cm})$. Thus, the fitting parameter with the Gaussian correlation function replaces the correlation length, corrects the imperfections of IEM, and allows using the same correlation function for all $r m s$ values.

\section{VALIDATION OF THE IEM CALIBRATION}

In order to validate the IEM calibration, Orgeval and Matera databases were used. Figure 6 illustrates the results provided by the IEM before and after calibration. In the calibrated version of the IEM, we used the Lopt2 given in the expression (2). Results show that the proposed semi-empirical calibration of the IEM provides improved results. The bias and the standard deviation of the error have decreased from $47.5 \mathrm{~dB}$ to $+0.15 \mathrm{~dB}$ (difference between IEM and SIR-C), and from $41.1 \mathrm{~dB}$ to $3.3 \mathrm{~dB}$, respectively.
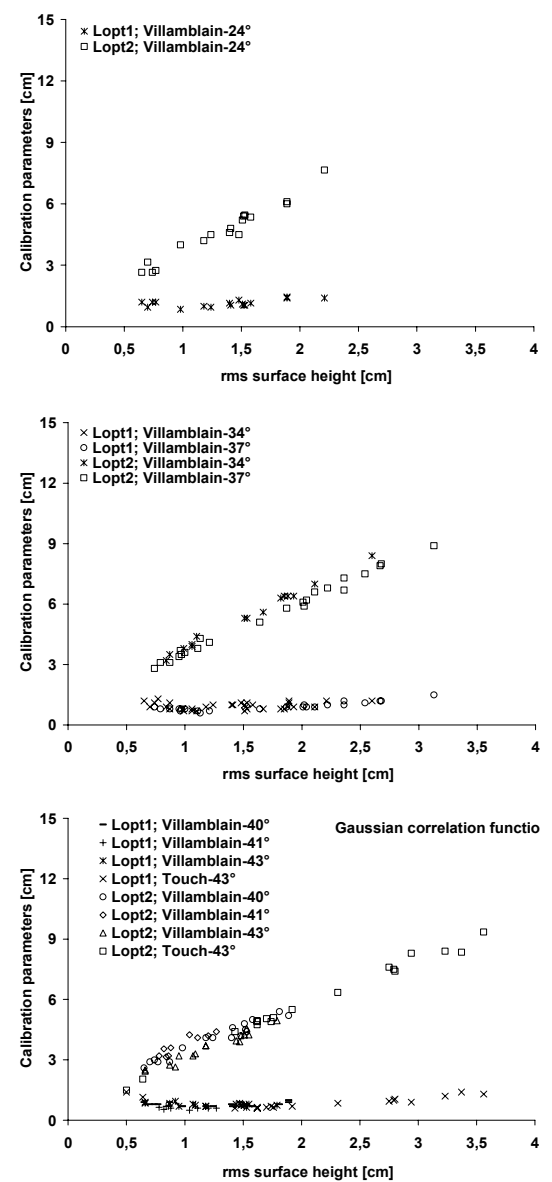

Fig. 4. Calibration parameters Lopt1 and Lopt2 as a function of $s$ (C-band, $\mathrm{HV}$ ), with Gaussian correlation function.

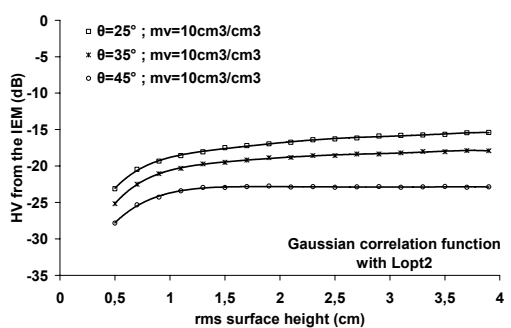

Fig. 5. IEM behaviour as a function of $r m s$ surface height, using the expression of Lopt2 (eq. (2)), frequency $=5.3 \mathrm{GHz}$, Clay $=30 \%$, Sand $=10 \%$.

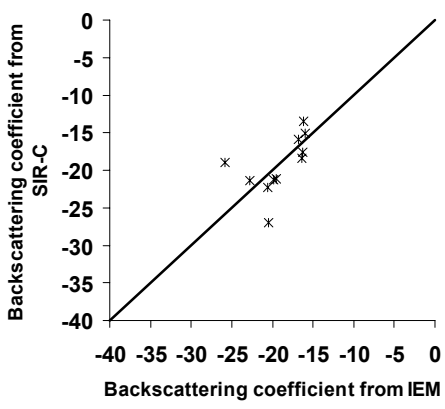


Fig. 6. Comparison between IEM using fitting parameter Lopt2 and SAR.

\section{CONCLUSIONS}

The characteristics of soil surface, mainly the moisture content and roughness, play an important role in hydrological studies. Synthetic Aperture Radar is a vital tool for measuring and mapping soil parameters. The estimation of soil parameters requires the use of backscattering models that are capable of reproducing a radar signal similar to that measured by SAR sensors.

The Integral Equation Model (IEM) is one of the models most widely used in inversion procedures for retrieving soil moisture and/or roughness parameters. However, numerous studies have observed a discrepancy between IEM simulations and SAR data. This discrepancy was related to the uncertainty of the correlation length measurements and to the model itself. The semi-empirical calibration of the IEM proposed in this study ensures better agreement between IEM and the SAR data. It consisted of finding a calibration parameter which replaces the inaccurate correlation length measurements and corrects the defects of model. This calibration was carried out using C-band radar configurations with different incidence angles $\left(24^{\circ}\right.$ to $\left.45.8^{\circ}\right)$ and $\mathrm{HV}$ polarization.

The results showed that the calibration parameter was found to be dependent on rms surface height and radar incidence angle. Moreover, the simulations produced by the calibrated IEM fit correctly SAR measurements (bias and standard deviation of the error were reduced). With this calibration, bare agricultural soils can be characterized by two surface parameters ( $r m s$ height and soil moisture) instead of four ( $r m s$ height, correlation length, correlation function, and soil moisture). Concerning the validity of the used approach, the fitting parameter allows correct estimation of the radar backscattering coefficient when the physical correlation length and rms surface height are correlated. In the future, it would be interesting to test the performances of this calibrated IEM version in using additional SAR configurations with incidence angles between $20^{\circ}$ and $45^{\circ}$.

With the results of this study and that of Baghdadi et al. [14], a semi empirical calibration of the IEM is now available for $\mathrm{HH}, \mathrm{HV}$, and VV polarizations. They should ensure an operational use of the IEM in the C-band SAR data inversion process (ERS-1/2, RADARSAT-1/2, ASAR, etc.).

\section{ACKNOWLEDGMENT}

This work was supported by the CEMAGREF (Agricultural and Environmental Engineering Research). The authors wish to thank ESA (European Space Agency) for kindly providing the SAR images within the framework of many projects (AOALO.361, AOLAO.3610, 351 ENVISAT/SAR). The authors wish to thank Francesco Mattia (ISSIA-CNR, BariItaly) for kindly providing Matera database published in [12].

\section{REFERENCES}

[1] F. T. Ulaby, P. P. Batlivala, and M. C., "Microwave backscatter dependence on surface roughness, soil moisture, and soil texture: Part I -
Bare soil,” IEEE Transactions on Geoscience and Remote Sensing, vol. GE-16, no. 4, pp. 286-295, 1978.

[2] S. Huang, L. Tsang, E. G. Njoku, and K. S.Chan, "Backscattering coefficients, coherent reflectivities, and emissivities of randomly rough soil surfaces at L-band for SMAP applications based on numerical solutions of Maxwell equations in three-dimensional simulations," IEEE Transactions on Geoscience and Remote Sensing, in press, doi:10.1109/TGRS.2010.2040748.

[3] M. Zribi, and M. Dechambre, "A new empirical model to retrieve soil moisture and roughness from C-band radar data," Remote Sensing of Environment, vol. 84, pp. 42-52, 2002.

[4] N. Baghdadi, N. Holah, and M. Zribi, "Soil moisture estimation using multi-incidence and multi-polarization ASAR SAR data,” International Journal of Remote Sensing, vol. 27, no. 10, pp. 1907-1920, 2006.

[5] Y. Oh, "Quantitative retrieval of soil moisture content and surface roughness from multipolarized radar observations of bare soil surfaces," IEEE Transactions on Geoscience and Remote Sensing, vol. 42, no 3, pp. 596-601, 2004.

[6] P. Dubois, J. Van Zyl, and T. Engman, "Measuring soil moisture with imaging radars," IEEE Transactions on Geoscience and Remote Sensing, vol. 33, no. 4, pp. 915-926, 1995.

[7] M. Zribi, O. Taconet, S. Le Hegarat-Mascle, D. Vidal-Madjar, C. Emblanch, C. Loumagne, and M. Normand, "Backscattering behavior and simulation: Comparison over bare soils using SIR-C/X-SAR and ERASME 1994 data over Orgeval," Remote Sensing of Environment, vol. 59, pp. 256-266, 1997.

[8] N. Baghdadi and M. Zribi,” Evaluation of Radar Backscatter Models IEM, OH and Dubois Using Experimental Observations,” International Journal of Remote Sensing, vol. 27, no. 18, pp. 3831-3852, 2006.

[9] A. K. Fung, Z. Li, and K. S. Chen, "Backscattering from a randomly rough dielectric surface,” IEEE Transactions on Geoscience and Remote Sensing, vol. 30, no 2, pp. 356-369, 1992.

[10] J. Shi, J. Wang, A. Y. Hsu, P. E. O'Neill, and E. T. Engman, "Estimation of bare surface soil moisture and surface roughness parameter using Lband SAR image data," IEEE Transactions on Geoscience and Remote Sensing, vol. 35 , no. 5 , pp. $1254-1266,1997$.

[11] R. Bindlish and A. P. Barros, "Multifrequency soil moisture inversion from SAR measurements with the use of IEM," Remote Sensing of Environment, vol. 71, pp. 67-88, 2002.

[12] F. Mattia, T. Le Toan, J. C. Souyris, G. De Carolis, N. Floury, F. Posa, and G. Pasquariello, "The effect of surface roughness on multifrequency polarimetric SAR data," IEEE Transactions on Geoscience and Remote Sensing, vol. 35, no 4, pp. 954-966, 1997.

[13] N. Baghdadi, I. Gherboudj, M. Zribi, M. Sahebi, F. Bonn, and C. King, "Semi-empirical calibration of the IEM backscattering model using radar images and moisture and roughness field measurements," International Journal of Remote Sensing, vol. 25, no. 18, pp. 3593-3623, 2004.

[14] N. Baghdadi, N. Holah, and M. Zribi, "Calibration of the Integral Equation Model for SAR data in C-band and $\mathrm{HH}$ and VV polarizations. International Journal of Remote Sensing, vol. 27, no. 4, pp. 805-816, 2006.

[15] E. Altese, O. Bolognani, and M. Mancini, "Retrieving soil moisture over bare soil from ERS1 synthetic aperture radar data: Sensitivity analysis based on a theoretical surface scattering model and field data," Water Resources Research, vol. 32, no 3, pp. 653-661, 1996.

[16] Y. Oh and Y. Kay, "Condition for precise measurement of soil surface roughness," IEEE Transactions on Geoscience and Remote Sensing, vol. 36, no 2, pp. 691-695, 1998.

[17] N. Baghdadi, Ph. Paillou, M. Davidson, G. Grandjean, and P. Dubois, "Relationship between profile length and roughness parameters for natural surfaces," International Journal of Remote Sensing, vol. 21, no 17, pp. 3375-3381, 2000.

[18] M. Hallikaïnen, F. Ulaby, F. Dobson, M. EL Rayes, and L. Wu, "Microwave dielectric behavior of wet soil. Part I: Empirical models and experimental observations," IEEE Transactions on Geoscience and Remote Sensing, vol. 23, pp. 25-34, 1985.

[19] M. W. J. Davidson, F. Mattia, G. Satalino, N. E. C. Verhoest, T. Le Toan, M. Bourgeaud, J. M. B. Louis, and E. Attema," Joint statistical properties of $r m s$ height and correlation length derived from multi-site 1$\mathrm{m}$ roughness measurements," IEEE Transactions on Geoscience and Remote Sensing, vol. 41, no 7, pp. 1651-1658, 2003. 
Manuscript received February 08, 2010; revised March 10 and April 19, 2010.

Corresponding

author:

N.

Baghdadi

(e-mail:

nicolas.baghdadi@teledetection.fr)

nicolas.baghdadi@teledetection.fr). 